\title{
Development of a Performance Calibration System for X-26 Tasers
}

\author{
David Dawson, Yasheng Maimaitijiang, Andy Adler \\ Systems and Computer Engineering \\ Carleton University \\ Ottawa, Canada \\ adler@sce.carleton.ca
}

\begin{abstract}
Conducted Energy Weapons (specifically the Taser) are being increasingly used by police in several countries, and have also been subject to significant media concern over the level of emissions and applicable safety standards. One issue has been the variability in electrical output between weapons, and of individual weapons over time. In order to address this issue, we present work to: 1) establish consensus on the appropriate electrical parameters to characterize a weapon's biomedical effects, and 2) the development and design of a portable test system to measure these parameters. A weapon is electrically connected to a calibrated dummy resistive load of $600 \mathrm{ohms}$ and fired for $5 \mathrm{~s}$ while the output voltage is measured and the parameters are subsequently calculated. This test system has been used to characterize 256 shots from 84 weapons over 8 test episodes spanning 16 months.
\end{abstract}

\section{Keywords- Taser ${ }^{\circledR}$, Conducted Energy Weapon, Calibration}

\section{INTRODUCTION}

In this paper we define a set of parameters to characterize the electrical parameters which of a conducted energy weapon (CEW), and present the design development and initial testing of a portable field test system to measure such parameters.

Conducted energy weapons (CEWs) apply electrical current to incapacitate the body of the target. The most widely used CEWs are the M26 and X26 models from Taser International [1]. These weapons work by firing two small darts (electrodes) attached to wires into the subject. Electronics in the weapon then send a pulsatile electrical signal to the subject across the wires. The electrical source is designed to be sufficiently rapid and give enough energy to initiate muscular incapacitation in the subject. At the same time, CEWs attempt to limit the applied energy to safe levels (where the level of safety has seen significant controversy). CEWs have been called "less-lethal" or "intermediate" weapons (the term "nonlethal" is now less used). These weapons have been adopted by many police forces in several countries, because they are seen to provide many advantages to police, primarily by providing the officer with an intermediate force option (less lethal than a ballistic weapon) which provides a significant stand-off distance (compared to the usage distance other weapons like batons and pepper spray).

The M26 was the earliest widely distributed Taser model, but has largely been replaced in service by the X26, which was first released in 2003. A small number of other CEWs are available including newly released models from Taser
International, and products from other manufacturers. We present work to characterize the behavior of the all pulse energy weapons, while we focus on characterization of the X26.

Although CEW's have been in use in Canada since 2001, very little scientific or public attention was devoted to this lessthan-lethal weapon before 2008. In the past ten years in Canada, less than 30 deaths occurred proximal to the use of CEW's. A review of public inquiries or judicial reviews of deaths was made [2]. In November 2007, an incident at the Vancouver International Airport resulted in the death of Robert Dziekanski [2,3]. A Taser had been used to subdue $\mathrm{Mr}$. Dzienkanski when police were not able to communicate effectively with him. His interactions with the police were recorded on a cellphone camera and broadcast widely. The large resulting media interest crystallized public fear and opinion about CEW's.

In this context several questions were raised about CEW's which we classify as relating to safety and policy. The safety questions relate to the level of risk to the CEW subject. This question has been studied from the point of view of electrical safety standards [4,5], animal models $[5,6,7]$, tests on police volunteers [8], and retrospective analysis of police custody records [9]. All such safety research is made difficult by two factors: 1) is very difficult to build an adequate model of an arrest scenario, and 2) the occurrence of negative outcomes is so low ( 1 to 6 per year in Canada) that the statistical significance of study results is low. However, at least one conclusion is clear; the potential risk of a CEW depends dramatically on the subject: stress, alcohol or drug use and underlying physiological conditions strongly correlate with negative outcomes. The policy questions relate to how decisions on CEW's were made, and the concern that it appears that most decisions on weapon usage, safety, performance, purchase and maintenance were made without independent testing or validation.

Recently, it has become clear that neither of these lines of questions can be satisfactorily answered without adequate characterization of CEW's. Characterization of a CEW's measures its electrical output to determine its electrical characteristics in order to determine: 1) the electrical output of a single weapon and how its output varies during usage and over time, 2) the variability across the population of CEW's in use, 3) out-of-tolerance modes and expected lifespan of the CEW. Such characterization is important for biomedical safety 
research, which has typically been based on simulations or artificial signals based on the manufacturer specified output $[10,11]$, or that from a small number of weapons [17]. In the case of the manufacturer specifications, these values represent average pulses, rather than the maxima. Characterization is important for policy questions in order to ensure weapon reliability, and to allow forensic examination of CEW's as part of the investigation of in-custody deaths. This situation may be compared to that with ballistic weapons, for which detailed inspection and weapon retirement criteria are well established. Mr. Braidwood [3] conducted a public enquiry into issues surrounding the use of CEW's and in particular, the Vancouver airport incident. Many of his nineteen recommendations in the preliminary report (Part 10) touch upon the characterization and verification of performance of CEW's.

Based on this requirement for characterization, during 2008 some Canadian police forces began a large-scale laboratory investigation of CEW's in their inventory to verify the performance of these weapons. There were significant rates of out-of-tolerance in some inventories of CEW's [12]. In this context, there was a clear need to clarify the characterization process for CEW's, so that laboratory or - in the future - field tests could measure the correct parameters and measure them in the correct way.

In the remainder of this paper, we present our work to define parameters to characterize the electrical parameters of a $\mathrm{CEW}$, and present the design development and initial testing of a portable field test system to measure such parameters.

\section{The ElectricAl OUtPUt Of THE X26 TASER}

The X26 Taser fires a sequence of electrical pulses into a target at a rate of approximately 20 pulses/s. By opening and inspecting a weapon which was scheduled for destruction, we determined that each pulse is generated internally by charging of three different capacitors from a battery. When the voltage reaches a threshold, a small gas discharge tube will ionize and conduct the current from the capacitors to the output terminals. If the weapon has an electrode cartridge attached, the propellant will fire the dart electrodes (attached to wires) toward the target. If the electrodes make contact and have a low resistance path between them, current will flow and the weapon is considered to be in "closed circuit" mode. Otherwise the "open circuit" voltage from the capacitors will be seen at the output with low current.

Using the equipment described in section IV, we measured the closed circuit voltage across a load resistance of $600 \Omega$. Two different time resolutions are shown, in order to clarify the individual pulses and pulse sequences. The three capacitors provide the output at different time constants, as can be seen in Fig. 1. The fastest time constant produces a rapid (5 ns) pulse which appears to be designed to create a "plasma arc" which can be used to conduct through clothing or anther offset between the electrodes and the skin. The other two capacitors produce output with frequencies of approximately $100 \mathrm{kHz}$ and $10 \mathrm{kHz}$. The choice of these two frequencies has not been made clear by the manufacturer; in older, M26 weapon, only the rapid, and one main output capacitor are present.
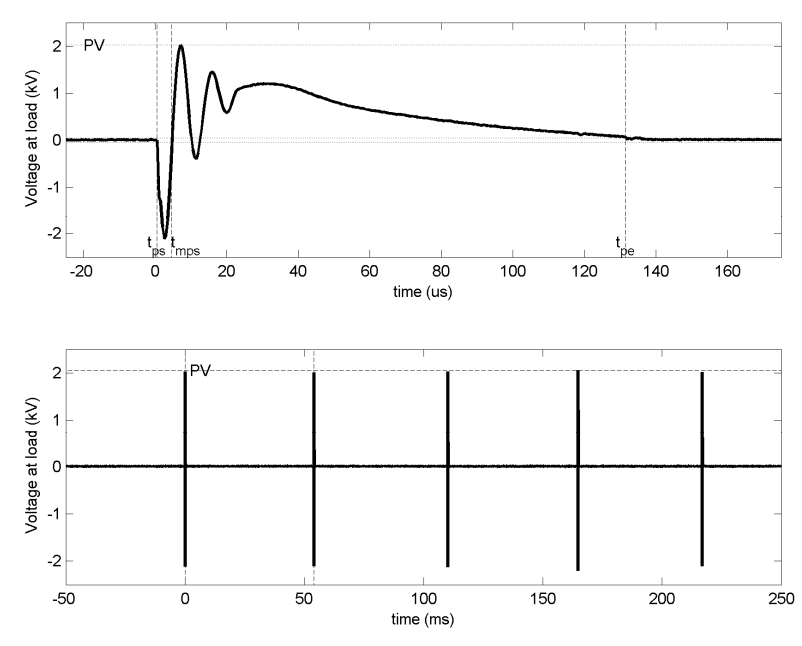

Figure 1: Taser X26 waveform: single pulse (top) and multiple pulses (bottom).

Based on these Taser output waveforms, we define electrical parameters to characterize the biomedical effects. At sufficiently high frequencies, the body is not sensitive to the detailed shape of the waveform, but rather to the cumulative effect. There is some disagreement about how to represent the effect. The main standard is the IEC electrical safety specification [13], which specifies the risk of ventricular fibrillation for three types of pulsatile currents, rectangular pulses, sinusoidal impulses, and capacitor discharges. A fibrillation energy, $F e$, and a fibrillation charge, $F q$, are defined as

$$
\begin{aligned}
& F e=\int I^{2} d t \\
& F q=\int|I| d t
\end{aligned}
$$

Based on these quantities, a graph of thresholds for ventricular fibrillation is given in Fig. 40 of [13]. For short pulses (less than $4 \mathrm{~ms}$ ), the graph and associated comments suggest that the probability of inducing ventricular fibrillation is largely a function of $F q$. Three thresholds are defined $\mathrm{C} 1$ (no fibrillation), $\mathrm{C} 2$ (5\% risk of fibrillation) and $\mathrm{C} 3$ (50\% risk). By fitting a curve to this figure, we calculate these thresholds as $F q(t)=F q(1 \mathrm{~ms}) \times t^{0.28}$ where $t$ is the pulse time in ms. The thresholds $F q(1 \mathrm{~ms})$ are $1350 \mathrm{uC}, 2700 \mathrm{uC}$, and $4320 \mathrm{uC}$, for $\mathrm{C} 1, \mathrm{C} 2$ and $\mathrm{C} 3$. If the Taser X26 pulse may be taken as roughly equivalent to a $0.1 \mathrm{~ms}$ rectangular pulse, then the $\mathrm{C} 1$ threshold is $710 \mathrm{uC}$.

Several caveats are made by the standard. First, "for impulses of short duration ..., ventricular fibrillation in general results only if the impulse falls within the vulnerable period of the cardiac cycle". However, since the X26 provides a series of pulses, it is reasonable to assume that at least one pulse will occur during the vulnerable period, and the worst case safety calculations must be taken. Second, these thresholds are a function of body mass, and must be scaled for body mass. Indeed, anecdotal evidence suggests that CEW's are less effective on larger and heavier subjects. Third, the calculations assume the absolute value of current. This means that the 
negative pulse phase from the X26 should be added to the charge. Finally, the threshold must be adjusted downward (by up to a factor of 10 or more) for sustained bursts of current.

Based on these considerations, it is important to define calculated parameters which describe the CEW pulse train in a way that is relevant to analysis according to the IEC standard [12] and other bioelectrical safety texts [14]. Fortunately, the parameters already defined by Taser international to characterize the X26 already capture this information, and only two additional parameters are required.

The parameters to describe each CEW pulse are as follows:

1. Peak Voltage (PV): peak of main phase voltage on a pulse see Fig. 1

2. Peak Current (PC): peak of main phase current on a pulse

3. Full Charge (FC): the integral of the absolute value of the current over the entire pulse train

4. Net Charge (NC): the integral of the current from $t_{\mathrm{mps}}$ to $t_{\mathrm{pe}}$. Any negative swings of the current

5. Pulse duration (PD): time from starting point of a waveform at an initial crossing of $\pm 50 \mathrm{~V}$ to end point at a pulse decrement to $\pm 50 \mathrm{~V}$ on a pulse

6. Interpulse time (IPT), the time from $t_{\mathrm{ps}}$ of one pulse to $t_{\mathrm{ps}}$ of the next pulse (see Fig. 1).

For these pulse parameters, it is necessary to define the timing of the pulses. The pulse start $\left(t_{\mathrm{ps}}\right)$ and end $\left(t_{\mathrm{pe}}\right)$ times are measured at the time which the voltage trace crosses $50 \mathrm{~V}$. The main phase start time $\left(t_{\mathrm{mps}}\right)$ is the time when the pulse crosses $50 \mathrm{~V}$ after the first negative phase. The value of $50 \mathrm{~V}$ is chosen as an estimate of the breakdown voltage of the skin. The exact value of the choice does not strongly affect the calculated timings.

Note that only the Net Charge parameter is related specifically to the waveform of the X26 Taser, since it requires a definition of a first downward pulse, which may not exist for all CEW devices.

One further parameter, the pulse repetition frequency (PRF) is calculated from the Interpulse time, as:

$$
P R F=\frac{1}{\text { mean }(I P T)}
$$

which may be calculated as follows

$($ Pulses -1$) /($ Total pulse time $)=($ Number of IPT $) /($ sum IPT $)$ $=1 /$ average IPT.

The PRF parameter matches the value published by Taser International. Note that in practice, there is significant variation in IPT for some weapons.

\section{DETERMINATION OF THE APPROPRIATE ELECTRICAL PARAMETERS}

In May 2009, we organized an International Workshop on CEW's at Carleton University in Ottawa, Canada [15]. International medical and engineering scientists, police officers and policy experts gathered to discuss the issues surrounding the use and performance of CEW's in police operations. The Canadian Association of Chiefs of Police and the Canadian Police Research Center produced papers and study documents which have formed valuable background for more diverse research interests. It was apparent that the measurement of the performance of CEW's was of significant interest to the range of experts studying the use of CEW`s. Questions considered were whether the weapons perform consistently, and if they perform in accordance with the manufacturer's specifications? Before 2008 there was no widespread testing of large numbers of CEW's in Canada or elsewhere.

A consensus of experts agreed in May 2009 [15] that the six parameters (section II) would adequately characterize the behavior of a CEW for the purpose of assessing performance for two criteria: 1) in terms of the biomedical effects, and 2) accordance with the manufacturer's performance criteria. Thus, to characterize a $5 \mathrm{~s}$ firing of a Taser X26, each parameter is calculated for each pulse. Based on approximately 20 pulses/s, this results in $5 \times 20 \times 6=600$ values per test. It was agreed that, while it is reasonable to store (electronically or on paper) such test results, it was necessary to provide summary values.

One area of slight disagreement was the appropriate way to summarize these parameters. The Taser international specification $[12,16]$ requires testing the average of the last eight pulses in the pulse train $(90-100$ pulses $)$. We suspect that this requirement was largely selected due to the capabilities of the Tektronics DPO series oscilloscopes which are specified in the document. While it was agreed that this "avg-8" approach was not unreasonable, it was clearly somewhat arbitrary. Instead, summary parameters should reflect the maxima, minima and average of each parameter (keeping the "avg-8" as an appropriate for testing to specifications).

The performance parameters set out by the manufacturer are summarized in Table 1.

Table 1. X26 performance parameters - the customer specification in 2009 [12]

\begin{tabular}{lc}
\hline Performance parameters & Value range \\
\hline PV (volts) & $1400-2520$ \\
PC (amps) & $2.3-4.2$ \\
NC ( $\mu$ Coulombs) & $85-125$ \\
PRF (pulses/s) & $16.5-20$ \\
PD $(\mu \mathrm{s})$ & $105-155$ \\
\hline
\end{tabular}

\section{DESIGN OF A TEST UNIT}

After experimenting with conventional analog signal capture with an oscilloscope and using an initial batch of 20 weapons, it was decided that insufficient data was available 
for meaningful biomedical research. Although verification of conformity with performance standards can be demonstrated with a lesser testing protocol, it is important to be able to capture all CEW pulses for larger investigations of biomedical effect from Taser emissions and provide some clues as to what causes out-of-tolerance and what are indicators of impending out-of-tolerance.

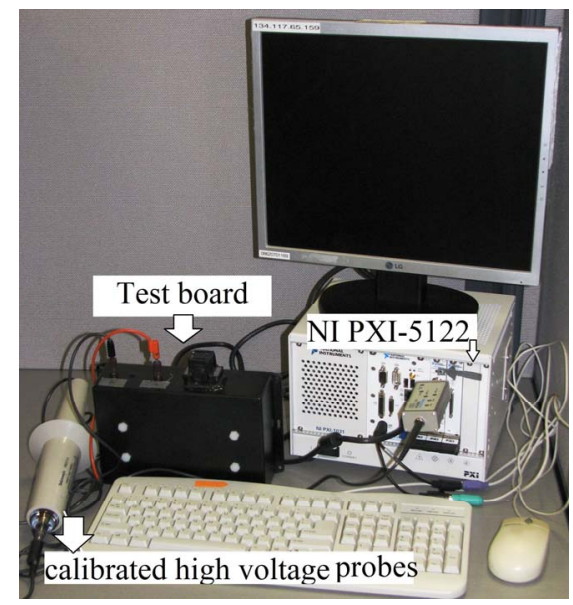

Figure 2. Prototype of performance calibration system for Taser X-26

Consequently we have developed a field-friendly and simple testing protocol using open source software (Octave) and modest equipments to test CEW's in the field. The performance evaluation system consists of Taser X-26 weapons, a National Instrument high-resolution digitizer (NI PXI-5122), calibrated high voltage probes, a calibrated dummy resistive load of 600 ohms and two test boards as shown in Fig. 2 and 3. The NI PXI-5122 contains an embedded control system that runs Octave. The weapon is electrically connected to the calibrated purely resistive load of $600 \mathrm{ohms}$.

Using this test system, a sufficient signal can be acquired in less than 15 seconds (including physical placement of a weapon in the mount, recording serial number, software version and battery capacity) and the analysis presented graphically in about 20 seconds per weapon. This protocol obviates the necessity to remove well-performing CEW's from service for an extended period of time and provides an instantaneous identification of poorly performing weapons which may require service. As well it digitizes the pulse train and preserves a raw data file so that interpulse activity can be observed and absolute maxima and minina derived. This procedure and testing equipment do not duplicate the manufacturer's suggested equipment and procedure but it provides sufficient information to determine performance in accordance with the manufacturer's expected limits (see Table 1).

Fig. 3 shows overview of performance calibration system for Taser X-26.

The procedure for performance calibration is as follows:

1. Acquire signal using a National Instrument highresolution digitizer.
2. Create and store data file, including serial number, battery capacity and software version in file title.

3. Analyze signal for absolute minima and maxima of V, I, $\mathrm{Q}, \mathrm{PRF}$, interpulse distance and pulse duration.

4. Present results 5 performance-indicating parameters based on average of last 8 pulses: V, I, Q, PRF, pulse duration in table 1 .

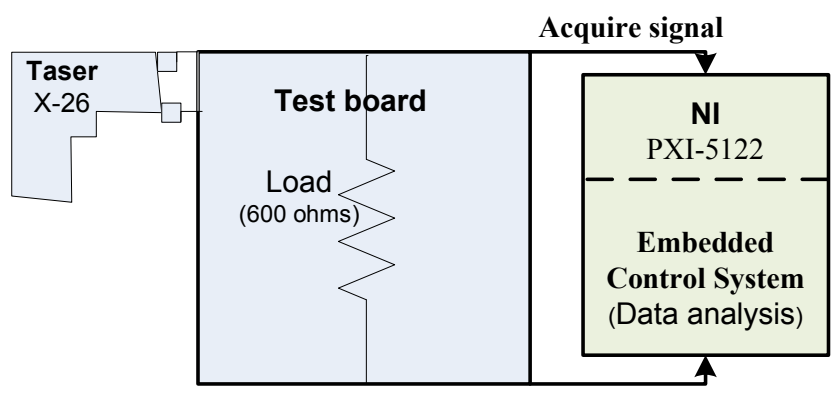

Figure 3. Overview of performance calibration system

It should be noted that creating and storing the raw data file at the time of testing allows researchers the opportunity to reinvestigate any aspect of the weapon's performance that may be of interest at a later date.

A sample waveform of one pulse indicates the shape of the pulsed waveform, the time, voltage and current amplitudes as shown in Fig. 4. The pulse train of 91 pulses indicates the relative amplitudes of voltage minima and maxima.

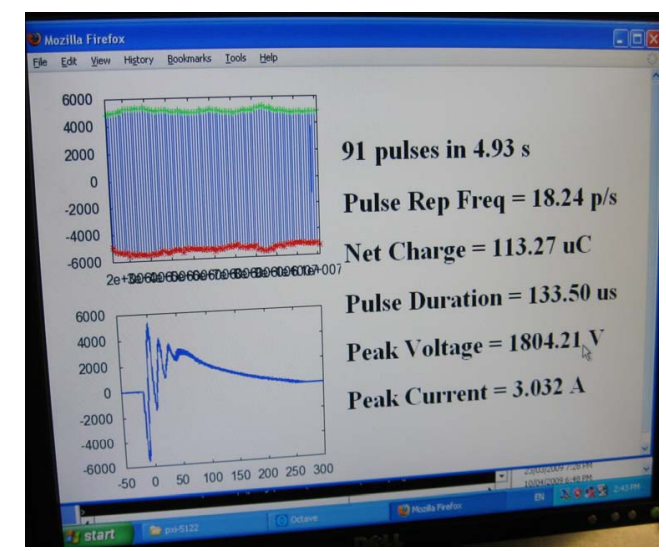

Figure 4. Sample waveshape of Taser X-26 emission

Using this system, we have measured performance data on individual weapons over time, individual weapons fired numerous times in succession and a large number of weapons of various ages in two different jurisdictions.

\section{TEST RESUlTS}

Using this system, the signals from 84 different weapons were subsequently sampled at a rate of $2 \mathrm{MHz}$ with a 14 bit digitizer using a National Instruments PXI-5122 digital sampling unit through a calibrated high voltage probe. To validate performance of the weapon in accordance with the parameters in Table 1 an average of the last 8 pulses was used 
for analysis. However, the entire data set from the approximately 5 second discharges (90-100 pulses) was preserved to compare these parameters with absolute maxima and minima over the pulse train and examine another parameter (interpulse time) that was not recognized to be a parameter of interest to the wider user community. Sample results from the analysis are found in Table 2.

For 82 pulses in $4.91 \mathrm{~s}, \mathrm{PRF}=16.49$ pps (pulses per second).

Table 2. Sample test results (Note: AVG-8 stands for the averaged values over the last eight pulses in a train of $90-100$ pulses.)

\begin{tabular}{lcccc}
\hline Summary & MAX & MIN & AVG & AVG-8 \\
\hline Net Charge (uC) & 112 & 100 & 106 & 106 \\
Full Charge (uC) & 116 & 103 & 110 & 110 \\
Pulse Duration (us) & 129 & 117 & 122 & 121 \\
Interpulse Time (ms) & 68 & 53 & 61 & 60 \\
Peak Voltage (V) & 1963 & 1741 & 1869 & 1869 \\
Peak Current (A) & 3.3 & 2.9 & 3.1 & 3.1 \\
\hline
\end{tabular}

A total of 256 raw data files from 84 different weapons were analyzed to determine overall out-of-tolerance rates. Significant out-of-tolerance rates were apparent in all but the newest of CEW's. The rate of out-of-tolerance of the weapons which we tested was $17 \%$.

Table 3. Out-of-tolerance rates from preliminary testing

\begin{tabular}{lcc}
\hline Testing period & Shots & $\begin{array}{c}\text { Out-of-tolerance } \\
\text { occurrences }\end{array}$ \\
\hline May 2009 & 3 & 0 \\
June 2009 & 38 & 8 \\
November 2009 & 77 & 24 \\
December 2009 & 33 & 10 \\
January 2010 & 85 & 0 \\
February 2010 & 20 & 3 \\
Total & 256 & 45 \\
\hline
\end{tabular}

A small number of our test results were compared with test results from testing done by other organizations and similar results were derived.

Performance variability:

In testing several weapons a number of times or the same weapon over an interval of several weeks, we have found a notunexpected consistency. When a weapon was out-of-tolerance one time, it remained out-of-tolerance every subsequent time it was fired or it continued to be out-of-tolerance several weeks later when re-tested. Police services who owned the weapons were very diligent to remove these weapons from service until their performance was verified as satisfactory.
Following analyses (Tables 4 and 5) are given as an example of their variable behavior of the Taser. Table 4 shows that all performance parameters are within the manufacturer's specified limits.

Table 4. Sample test results for in-tolerance Taser

\begin{tabular}{lcccc}
\hline Summary & MAX & MIN & AVG & AVG-8 \\
\hline Net Charge (uC) & 119 & 105 & 111 & 109 \\
Full Charge (uC) & 123 & 108 & 115 & 113 \\
Pulse Duration (us) & 131 & 116 & 128 & 126 \\
Interpulse Time (ms) & 62 & 49 & 54 & 55 \\
Peak Voltage (V) & 2094 & 1861 & 1938 & 1923 \\
Peak Current (A) & 3.52 & 3.13 & 3.26 & 3.23 \\
\hline
\end{tabular}

On the other hand, the following test report on a test of a weapon as shown in the Table 5 indicates two parameters which are outside the limits specified by the manufacturer.

Table 5. Sample test results for an out-of-tolerance Taser

\begin{tabular}{lcccc}
\hline Summary & MAX & MIN & AVG & AVG-8 \\
\hline Net Charge (uC) & 129 & 115.7 & 124.2 & 125.3 \\
Full Charge (uC) & 133 & 119 & 129 & 130 \\
Pulse Duration (us) & 152 & 123 & 136 & 135 \\
Interpulse Time (ms) & 108 & 62 & 70 & 72 \\
Peak Voltage (V) & 2234 & 1988 & 2160 & 2183 \\
Peak Current (A) & 3.75 & 3.34 & 3.63 & 3.67 \\
\hline
\end{tabular}

In this case the pulse repetition rate (frequency) is 14.22 which is below the manufacturer's lower acceptable threshold of 16.49 pulses per second. Similarly the Net Charge is slightly in excess of the $125 \mu \mathrm{C}$ upper limit of net charge.

\section{DISCUSSION AND CONCLUSION}

In this paper we have defined a set of parameters to characterize the electrical parameters of a CEW, and demonstrated the design and testing of a portable field test system to measure these parameters based on 256 shots from 84 weapons over 8 test episodes spanning 16 months.

Most of the out-of-tolerance noted in our testing were triggered by a pulse repetition rate that was too slow. While the manufacturer indicated in verbal testimony to the House of Commons Standing Committee of Justice that pulse rates of greater than $14 \mathrm{pps}$ will induce neuromuscular incapacitation, we found that most of the out-of-tolerance conditions involved pulse rates between 12 and 16.5 pps. These out-of-tolerance conditions were audible to the human ear. Replacement of the power source (Digital Power Magazine) restored most of these weapons to an in-tolerance state. However, there were a small number of weapons which were not serviceable at all. They either produced no emissions or pulse rates which could not be brought within tolerance. 
A brief analysis of out-of-tolerance conditions follows in Table 6. In some cases two or more out-of-tolerance parameters presented on the same weapon and the same shot episode.

Table 6. Occurrence of out-of-tolerance parameters

\begin{tabular}{lc}
\hline $\begin{array}{l}\text { Out-of-tolerance } \\
\text { parameters }\end{array}$ & Occurrences \\
\hline $\mathrm{V}_{\max }>\mathrm{V}_{\text {manufacturer }}$ & 5 \\
$\mathrm{I}_{\max }>\mathrm{I}_{\text {manufacturer }}$ & 5 \\
$\mathrm{Q}_{\text {net }}>\mathrm{Q}_{\text {manufacturer }}=125 \mu \mathrm{C}$ & 17 \\
Pulse duration $>155 \mu \mathrm{s}$ & 3 \\
$\mathrm{PRF}<16.5$ pps & 32 \\
\hline
\end{tabular}

Regular performance testing of CEW's:

Because no routine or regular performance testing of CEW's currently exists for the thousands of weapons in service in Canada, police services do not have any guidance for life cycle planning for these weapons. They do not know how long they will last or whether the capital cost is a value-for-money investment. Neither do they have a regular means of verifying the performance of the weapons over time.

A case must be made for regular testing in accordance with a generally accepted testing protocol. That is not to say that the exact equipment and configuration that the manufacturer uses to verify performance after manufacture or during warranty work is the only one or the best one. Test equipment should provide a traceable calibration to acceptable lab standards for the equipment being used. Minimum technical requirements for a signal sampling are: $200 \mathrm{MHz}$ bandwidth, $2 \mathrm{MHz}$ sampling rate, 14 bit quantization, $600 \mathrm{ohms}$ and 10 watts of purely resistive load. Higher sampling rates and lesser quantization ( 8 bits) are also acceptable.

Any test protocol should acquire enough information about the parameters of the signal to make the data useful in a larger biomedical context.

The test protocol should include a thorough visual inspection and a verification of the mechanical components. Tombstone information such as serial number, beginning battery capacity and software version should be recorded. A full five seconds of signal discharge should be digitized to produce a data set which would yield performance information as well as other information such as absolute minima and maxima in the pulse train, among other things such as interpulse distance and averages over all the pulses in the $5 \mathrm{~s}$ pulse train.

Interpulse spacing and fidelity of the waveshape are important components of the additional data necessary. As well, the test protocol should establish a bench mark indication of how the weapon performs should it be necessary to do a forensic examination of the $\mathrm{CEW}$ in an ex post facto situation. All test results should be tabulated and displayed beside the manufacturer's performance values (see Table 1).

Test results showed that older weapons perform less well than newer ones and $17 \%$ of weapons in our tests remained out-of-tolerance mostly with respect to low PRF. Routine testing is strongly indicated for all CEW's with the same diligence as is currently applied to ballistic weapons.

This test system characterizes the CEW (Taser X26) well, and can contribute a novel and cost-effective solution to the verification and performance evaluation of the Taser.

\section{REFERENCES}

[1] TASER International. TASER series electronic control device specification. [World-Wide Web Page]. 15 May 2007. http://www.taser.com/SiteCollectionDocuments/Controlled $\% 20$ Documents/Spec\%20Sheets/RD-SPEC-C2-001_A.pdf.

[2] G. Breitkreuz, "Study of the Conductive Energy Weapon-Taser", Report of the Standing Committee on Public Safety and National Security, Standing Order 108(2), Canada, June 2008.

[3] T.R. Braidwood, "Braidwood Commission on Conducted Energy Weapon use", Braidwood Commissions of Inquiry, Vancouver, Printed Rep. 7550003670, May 2008. http://www.braidwoodinquiry.ca/report/.

[4] P. Savard, R. Walter, A. Dennis, "Analysis of the quality and safety of the Taser X26 devices tested for Radio-Canada / Canadian Broadcasting Corporation," National Technical Systems, Test Report 41196-08, 2008.

[5] J.R. Jauchem, "An animal model to investigate effectiveness and safety of electronic control devices (including TASER devices)," J Forensic Sci. Vol. 55 Issue 2, p. 521-526, February 2010.

[6] A. J. Dennis, D. J. Valentino, R. J. Walter, K. K. Nagy, J. Winners, F. Bokhari, D. E.Wiley, K. T. Joseph, and R. R. Roberts, "Acute effects of TASER X26 discharges in a swine model,” J. Trauma, vol. 63, pp. 581590, 2007.

[7] A.O. Esquivel, E.J. Dawe, J.A. Sala-Mercado, R.L. Hammond, C.A. Bir, "The physiologic effects of a conducted electrical weapon in swine," Ann Emerg Med. 50:576-83, 2007.

[8] J. Kleinig, "Ethical constraints on Taser use by police," A Journal of Policy and Practice, Vol. 1, No. 3, p. 284-292, 2007.

[9] C. Mesloh, M. Henych, R. Wolf, "Less lethal weapon effectiveness, use of force, and suspect \& officer injuries: a five-year analysis," Law Enforcement Executive Forum, Vol. 5, No. 5, p. 67-79, 2005.

[10] C. Mesloh, M. Henych, L.F. Thompson, R. Wolf, "A Qualitative \& quantitative analysis of conducted energy devices: TASER X26 vs. Stinger S200," A report to the National Institute of Justice, United States Department of Justice, Document No.:222769, March 2008.

[11] D.I Wilkinson, "Supplement to HOSDB evaluations of Taser devices," Home Office Scientific Development Branch,United Kingdom, Publication No. 64/06, November 2006.

[12] I.P.W. Sinclair, "Test Results for the X26 Conducted Energy Weapon (CEW) in accordance with Taser International Device Specifications," MPB Technologies - Electronics Test Centre, Report No.: A85R9006/9031/9059B1, 2009.

[13] IEC/TS 60479-2, "Effects of current on human beings and livestock Part 2: Special aspect," Ed. 3.0: May 2007.

[14] J.P. Reilly, Applied bioelectricity: from electrical stimulation to electropathology, Springer-V, ISBN: 0387984070, 1998.

[15] A. Adler, Workshop on Conducted Energy Weapons, Carleton University, Ottawa, Ontario, May 2009. http://www.sce.carleton.ca/faculty/adler/talks/2009/adler-CEWWorkshop.pdf.

[16] S. Palmer, "Testing of Conducted Energy Weapons," Canadian Police Research Centre, OPCC file 2587, April 2008.

[17] F. Zalac, "Some tested Tasers fire stronger current than company says: CBC/Radio-Canada probe," CBC/Radio-Canada, December 4, 2008. Available: http://www.cbc.ca/canada/story/2008/12/04/taser-tests.html [Accessed April 5, 2010]. 